\title{
CCL21 and IP-10 as blood biomarkers for pulmonary involvement in systemic lupus erythematosus patients
}

\author{
B Odler ${ }^{1}$, A Bikov ${ }^{1}$, J Streizig ${ }^{1}$, C Balogh ${ }^{1}$, E Kiss $^{2}$, K Vincze $^{1}$, I Barta $^{3}$, I Horváth ${ }^{1,3}$ and V Müller ${ }^{1}$ \\ ${ }^{1}$ Department of Pulmonology, Semmelweis University, Budapest, Hungary; ${ }^{2}$ National Institute of Rheumatology and Physiotherapy, Budapest, \\ Hungary; and ${ }^{3}$ Department of Pathophysiology, National Korányi Institute of TB and Pulmonology, Budapest, Hungary
}

\begin{abstract}
Biomarkers for pulmonary manifestations in systemic lupus erythematosus (SLE) are missing. Plasma samples of nine SLE patients with known pulmonary involvement ( $\mathrm{SLE}_{\text {pulm }}$ ) and nine SLE patients without pulmonary involvement (SLE) were tested by multiplex microarray analysis for various cyto- and chemokines. Significantly decreased lung function paramters for forced vital capacity (FVC), total lung capacity (TLC), diffusion capacity for carbon monoxide $\left(\mathrm{DL}_{\mathrm{CO}}\right)$ and diffusion of $\mathrm{CO}$ corrected on lung volume $\left(\mathrm{KL}_{\mathrm{CO}}\right)$ were observed in SLE $_{\text {pulm }}$ as compared to SLE patients. CC chemokine ligand 21 (CCL21) and interferon

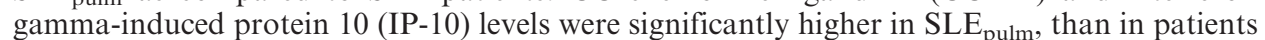
without pulmonary manifestations. CCL21 correlated negatively with $\mathrm{DL}_{\mathrm{CO}}(r=-0.73$; $p<0.01)$ and $\mathrm{KL}_{\mathrm{CO}}(r=-0.62 ; p<0.01)$, while IP-10 with $\mathrm{FVC}$ and forced expiratory volume one second. Receiver Operating Characteristics (ROC) analysis confirmed high sensitivity and specificity for the separation of SLE patients with and without pulmonary involvement for the chemokines CCL21 (Area Under Curve (AUC): 0.85; sensitivity\%: 88.90; specificity\%: 75.00; $p<0.01$ ) and IP-10 (AUC: 0.82; sensitivity\%: 66.67, specificity\%: 100; $p<0.01$ ). Pleuropulmonary manifestations in SLE patients associated with lung functional and $\mathrm{DL}_{\mathrm{CO}} / \mathrm{KL}_{\mathrm{CO}}$ changes and were associated with significant increase in CCL21 and IP-10. These chemokines might serve as potential biomarkers of lung involvement in SLE patients. Lupus (2016) 0, 1-8.
\end{abstract}

Key words: Systemic lupus erythematosus; immunoassay; diffusion capacity; lung function; CCL21; IP-10

\section{Introduction}

Systemic lupus erythematosus (SLE) is a systemic autoimmune disease that can affect almost any organ system. The disease is characterised by an autoantibody response to nuclear and cytoplasmic antigens. ${ }^{1}$ SLE predominantly affects females with onset during child-bearing age, with a female-male ratio of 9:1 and a higher prevalence in Hispanics, African-Americans and Afro-Caribbeans as compared to the Caucasian population. ${ }^{2,3}$ Its presentation and course are highly variable, ranging from inactive to fulminant. ${ }^{4}$

Correspondence to: Veronika Müller, Semmelweis University, Department of Pulmonology, Diós árok u. 1/c, H-1125 Budapest, Hungary.

Email: muller.veronika@med.semmelweis-univ.hu

Received 28 April 2016; accepted 16 August 2016
Involvement of the respiratory tract is more frequent in SLE patients, as previously assumed and also confirmed in our previous study. ${ }^{5}$ The prevalence ranges from 4.9 to $50 \%$ in the adult population, while it is $5-67 \%$ in juvenile SLE according to previous studies, depending upon whether only symptomatic patients or detailed pulmonary examinations are included. ${ }^{6-8}$ The disease might present with the first signs of pulmonary symptoms in about $4-5 \%$ of patients and lungs are frequently involved during the disease course. ${ }^{9}$ Depending on the affected area, pulmonary manifestations include pleuritis, interstitial lung disease (ILD), airway disease, vascular or musculoskeletal involvement. ${ }^{10}$ Moreover, some other conditions, such as pulmonary embolism, complementmediated leukoaggregation, infections or the life threatening alveolar haemorrhage can accompany the disorder. ${ }^{11}$

The role of inflammatory cyto- and chemokines is well known in the pathogenesis of SLE, however 
the results are controversial and suggest that combinations of different biomarker measurements could be useful to follow up disease activity or organ involvement. ${ }^{12}$ In terms of lung involvement only a few studies are available, suggesting that some of these markers can be elevated in blood and exhaled breath condensate of SLE patients with pulmonary manifestations. ${ }^{13,14}$ Previous studies reported interleukin (IL)-6, IL-8, interferon- $\gamma$ $(\mathrm{INF}-\gamma)$ and tumor necrosis factor- $\alpha(\mathrm{TNF}-\alpha)$ as possible markers for pulmonary manifestation in SLE patients. ${ }^{13,15}$ However, these markers are not specific enough to identify the pulmonary manifestations, as their levels are elevated in many pathophysiological conditions.

Despite the potentially high prevalence of lung complications in SLE, only a limited number of studies assessed risk factors that might predict pulmonary manifestations or biomarkers associated with lung involvement. Nevertheless, no previous studies specifically investigated the possible role of cyto- and chemokines in SLE patients with and without lung manifestations. Therefore, we chose 10 possible cyto- and chemokines based on the literature background of their possible role in the pathogenesis of SLE or diseases of the lung. Our aim was to test and identify potential biomarkers for pulmonary manifestations and to examine their relationship with clinical parameters in this rare, but high risk, group of SLE patients.

\section{Methods}

\section{Patient recruitment}

Healthy control subjects $(n=9)$ were all volunteers. Nine SLE patients with known lung manifestations $\left(\mathrm{SLE}_{\text {pulm }}\right)$ and nine SLE patients without lung involvement (SLE) were enrolled into the study. SLE patients were recruited consecutively at the outpatient clinic of the Department of Pulmonology, Semmelweis University, having been referred to our clinic for pleuropulmonary involvement screening by the National Department of Rheumatology and Physiotherapy. All patients fulfilled the American College of Rheumatology (ACR) criteria for the classification of SLE. ${ }^{16}$ Patients with infections six weeks prior to the study were excluded. All cases were of Caucasian ethnic origin.

Written informed consent was obtained from all subjects, and our study was reviewed and approved by the Ethical Committee of the National Institute of Rheumatology and Physiotherapy (reference number: ad.223/2014/EKU). The study adhered to the tenets of the most recent revision of the Declaration of Helsinki.

\section{Study design}

Venous blood was collected into EDTA tubes from all subjects, centrifuged at $1500 \mathrm{r} / \mathrm{min}$ at $4^{\circ} \mathrm{C}$ within two hours for routine haematological analysis and the remaining sample was stored at $-80^{\circ} \mathrm{C}$ until cytokine and chemokine measurements. Organ manifestations and actual medications were recorded from medical files. Patients were asked about their respiratory symptoms, underwent physical examination, chest X-ray, electrocardiography and routine laboratory analysis. Arterialised capillary blood gases, $\mathrm{pH}$ and bicarbonate levels were analysed in all patients at rest in room air (Roche Cobas b 221 Blood Gas system ${ }^{\circledR}$, Roche Diagnostics Corporation, USA).

Spirometry [Forced vital capacity (FVC), forced expiratory volume $1\left(\mathrm{FEV}_{1}\right), \mathrm{FEV}_{1} / \mathrm{FVC}$, forced expiratory flow between $25 \%$ and $75 \%$ of $\mathrm{FVC}$ (FEF25-75), total lung capacity (TLC), residual volume (RV), thoracic gas volume (TGV) and airway resistance (Raw)] was measured by means of electronic spirometer (PDD-301/s, Piston, Budapest, Hungary) according to the American Thoracic Society guidelines. ${ }^{17}$ Three technically acceptable manoeuvres were performed, and the best of them was evaluated. Diffusion capacity for carbon monoxide $(\mathrm{CO})\left(\mathrm{DL}_{\mathrm{CO}}\right)$ and diffusion of $\mathrm{CO}$ corrected on lung volume $\left(\mathrm{KL}_{\mathrm{CO}}\right)$ were measured with single breath method (PDD-301/s, Piston, Budapest, Hungary). Pulmonary function variables were expressed as a percentage of predicted values.

\section{Cytokine antibody array and quantification}

Custom made human Cytokine Antibody Array Kits (RayBiotech Inc., Norcross, GA, USA) were used to determine plasma cyto- and chemokine levels. Microarray analysis was carried out according to the recommendations of the array manufacturer. The following factors were measured in parallel on a single array per patient: 4BB-1; interferon gamma-induced protein 10 (IP-10); interleukin-12, p70 (IL12p70); interleukin-17 (IL-17); CC chemokine ligand 14a (CCL14); CC chemokine ligand 21 (CCL21); CC chemokine ligand 28 (CCL28); monocyte chemotactic protein 1 (MCP1); monocyte chemotactic protein 4 (MCP-4); interferon-inducible $\mathrm{T}$ cell alpha chemoattractant (I-TAC). Signal intensities were recorded and sorted using a chemiluminescence imaging system 
(Syngene Chemigenius 2 and GeneTools, Synoptics Ltd. Cambridge, UK). Background corrected raw intensities were used for analysis.

\section{Statistical analysis}

Statistical analyses were performed applying the following software: Graph Pad Prism software 5.0 (GraphPad Software, La Jolla, CA, USA). The normality distribution of the data was assessed by the Kolmogorov-Smirnoff test. Clinical data between SLE patient groups and controls were analyzed by one-way ANOVA test if the data showed normal distribution and Kruskal-Wallis U test followed by Dunn's post hoc test if the data were not normally distributed. The relationship between clinical parameters and microassay results was determined with Spearman rank correlation when the data showed normal distribution and Pearson rank correlation with not normally distributed data. Area Under Curve (AUC) values of Receiver Operating Characteristics (ROC) curves were calculated using standard methods and data are presented as AUC ROC $(95 \%$ CI). For all statistical analyses $p<0.05$ was considered statistically significant. Unless otherwise specified, data are reported as mean $\pm \mathrm{SEM}$ for continuous variables and as number (percent) for categorical variables.

\section{Results}

\section{Clinical characteristics}

The study had a cross sectional design. Baseline patient characteristics are summarised in Table 1.

There was no statistically significant difference in mean age $(50.48 \pm 2.69$ versus $38.23 \pm 5.46$ years; $p>0.05$ ) between the SLE and SLE $_{\text {pulm groups. }}$ Patients without lung manifestations had higher body mass index (BMI) than SLE $_{\text {pulm }}(27.29 \pm$ 2.06 versus $\left.20.64 \pm 1.52 \mathrm{~kg} / \mathrm{m}^{2} ; p<0.01\right)$. Fortyfour percent (4 out of 9) of $\mathrm{SLE}_{\text {pulm }}$ and SLE patients were smokers. Pack/year exposures were comparable in the two groups. Eighty-eight percent of the $\mathrm{SLE}_{\text {pulm }}$ patients were on corticosteroid therapy and had higher doses than the $44 \%$ of SLE patients, as summarised in Table 1.

Table 1 Patient characteristics of systemic lupus erythematosus patients with and without lung manifestations

\begin{tabular}{|c|c|c|c|}
\hline & $\begin{array}{l}\text { SLE patients without } \\
\text { lung manifestations }\end{array}$ & $\begin{array}{l}\text { SLE patients with } \\
\text { lung manifestations }\end{array}$ & $\mathrm{p}$-values \\
\hline Number of patients $(n)$ & 9 & 9 & - \\
\hline Female/male & $9 / 0$ & $7 / 2$ & - \\
\hline Age & $50.48 \pm 2.69$ & $38.23 \pm 5.46$ & 0.0615 \\
\hline mean [range] & {$[33-60]$} & {$[17-65]$} & \\
\hline BMI $\left(\mathrm{kg} / \mathrm{m}^{2}\right)$ & $27.29 \pm 2.06$ & $20.64 \pm 1.52$ & 0.0142 \\
\hline Smoker $n(\%)$ & $4(44)$ & $4(44)$ & - \\
\hline \multicolumn{4}{|l|}{ Pulmonary symptoms } \\
\hline Cough $n(\%)$ & $4(44)$ & $5(55)$ & - \\
\hline Dyspnea $n(\%)$ & $6(67)$ & $4(44)$ & \\
\hline Chest pain $n(\%)$ & $3(33)$ & $2(22)$ & \\
\hline Methylprednisolone (all), $n(\%)$ & $4(44)$ & $8(88)$ & - \\
\hline $4 \mathrm{mg} /$ day, $n$ & 2 & 2 & \\
\hline $8 \mathrm{mg} /$ day, $n$ & 2 & 4 & \\
\hline $16 \mathrm{mg} /$ day, $n$ & 0 & 2 & \\
\hline \multicolumn{4}{|l|}{ ACR criteria } \\
\hline Malar rash $n(\%)$ & $4(44)$ & $4(44)$ & - \\
\hline CDLE $n(\%)$ & $0(0)$ & $1(11)$ & \\
\hline Photosensitivity $n(\%)$ & $8(88)$ & $3(33)$ & \\
\hline Mucosal lesions $n(\%)$ & $3(33)$ & $0(0)$ & \\
\hline Arthritis $n(\%)$ & 7 (77) & $8(88)$ & \\
\hline Serositis $n(\%)$ & $3(33)$ & $5(55)$ & \\
\hline Kidney $n(\%)$ & $2(22)$ & $3(33)$ & \\
\hline CNS $n(\%)$ & $3(33)$ & $3(33)$ & \\
\hline Hematological $n(\%)$ & $6(66)$ & $5(55)$ & \\
\hline Immunological $n(\%)$ & $5(55)$ & $6(66)$ & \\
\hline ANA positivity $n(\%)$ & $8(88)$ & $9(100)$ & \\
\hline
\end{tabular}

Data expressed as mean \pm SEM if not stated otherwise.

$\mathrm{BMI}=$ body mass index; $\mathrm{ACR}=$ American College of Rheumatology; $\mathrm{ANA}=$ antinuclear antibody $\mathrm{CDLE}=$ chronic discoid lupus erythematosus; $\mathrm{CNS}=$ central nervous system. 
Table 2 Appearance of lung involvement in systemic lupus erythematosus patients and immunosuppressive therapy used

\begin{tabular}{|c|c|c|c|c|c|c|}
\hline $\begin{array}{l}\text { Patient ID } \\
\text { number }\end{array}$ & Gender & $A g e^{a}$ & $\begin{array}{l}\text { Lung function } \\
\text { abnormality }\end{array}$ & $\begin{array}{l}\text { Diffusion } \\
\text { capacity }^{b}\end{array}$ & $\begin{array}{l}\text { Lung } \\
\text { involvement }^{c}\end{array}$ & $\begin{array}{l}\text { Systemic } \\
\text { immunosuppressive therapy }^{d}\end{array}$ \\
\hline SLE 1 & Female & 59 & Restrictive & $\downarrow \downarrow$ & ILD + bronchiectasia & MP \\
\hline SLE 2 & Female & 41 & Mixed & $\downarrow \downarrow$ & $\mathrm{ILD}+\mathrm{SAI}$ & MP \\
\hline SLE 3 & Female & 23 & Restrictive & - & SLS & MP, AZA \\
\hline SLE 4 & Female & 40 & Obstructive & $\downarrow$ & SAI & MP \\
\hline SLE 5 & Male & 65 & - & $\downarrow \downarrow$ & ILD + emphysema & - \\
\hline SLE 6 & Male & 40 & Mixed & $\downarrow \downarrow$ & ILD + SAI & MP \\
\hline SLE 7 & Female & 17 & Restrictive & $\downarrow \downarrow$ & ILD & $\mathrm{MP}, \mathrm{CSA}$ \\
\hline SLE 8 & Female & 36 & Restrictive & $\downarrow \downarrow$ & ILD & MP, AZA \\
\hline SLE 9 & Female & 19 & Restrictive & $\downarrow \downarrow$ & ILD & MP \\
\hline
\end{tabular}

${ }^{\text {a }}$ Mean age at the time of the recruitment into the study.

${ }^{\mathrm{b}} \downarrow=$ decreased; $\downarrow \downarrow=$ severely decreased.

${ }^{\mathrm{c}} \mathrm{ILD}=$ interstitial lung disease; SAI = small airway involvement; SLS = shrinking lung syndrome

${ }^{\mathrm{d}} \mathrm{MP}=$ Methylprednisolone; AZA = Azathioprine; $\mathrm{CSA}=$ Cyclosporine A; MTX $=$ Methotrexate.

Pulmonary symptoms did not differ significantly in $\mathrm{SLE}_{\text {pulm }}$ patients as compared to SLE patients. In both groups, dyspnea and cough were the leading respiratory complaints. Types of pulmonary involvement(s) of each individual in the SLE pulm group are listed in Table 2. Interstitial lung disease (ILD) and small airway involvement were the most common lung involvements, while one patient had shrinking lung syndrome (SLS) without diffusion capacity changes. Moreover, only one patient had bronchiectasis and one other was diagnosed with concomitant emphysema.

The results of lung function and diffusion parameters for the respective SLE groups are given in Table 3. The mean values of lung function parameters were in the normal range in SLE patients, however FVC $(99.00 \pm 2.75$ versus $79.89 \pm 7.26 \%$ predicted; $p=0.02)$ and TLC $(93.22 \pm 3.40$ versus $76.89 \pm 3.37 \%$ predicted; $p<0.01)$ were decreased in the $\mathrm{SLE}_{\text {pulm }}$ patients. In addition, decreased values of diffusion parameters were observed in $\mathrm{SLE}_{\text {pulm }}$ patients including $\mathrm{DL}_{\mathrm{CO}}(90.56 \pm 6.79$ versus $60.75 \pm 5.18 \%$ predicted; $p<0.01)$ and $\mathrm{KL}_{\mathrm{CO}} \quad(72.78 \pm 4.71$ versus $60.25 \pm 3.69 \%$ predicted; $p=0.06$ ). Blood gas parameters did not differ in the study groups.

\section{Comparison of chemo- and cytokine levels between $S L E_{\text {pulm }}$ and SLE patients}

In several plasma samples the levels of 4BB-1, IL12p70, IL-17, CCL28, MCP-4 and I-TAC were below the detection limit of the array, therefore only data obtained for the remaining factors, CCL21, IP-10, MCP-1 and CCL14, were used in the statistical analysis.

As presented in Figure 1, the chemiluminescent intensity of CCL21 was significantly higher in
SLE $_{\text {pulm }}$ than in patients without lung involvement; however, no significant difference to the control group has been identified $(4949 \pm 1481$ versus $1272 \pm 236.1$ versus $1581 \pm 382.1 \quad \mathrm{U} ; \quad p<0.05$; Figure 1(a)). A significantly increased chemiluminescent intensity was detected for circulating IP-10 in the SLE $_{\text {pulm }}$ group as compared to SLE patients and healthy volunteers $(5923 \pm 1278$ versus $5759 \pm 926$ versus $10,762 \pm 1584 \mathrm{U} ; \quad p<0.05$; respectively; Figure 1(b)). In the case of MCP-1, the difference between the SLE study groups has not reached statistical significance; nevertheless, a tendency to an elevated level in the SLE $_{\text {pulm }}$ group was observed. However, in comparison with the control group, the $\mathrm{SLE}_{\text {pulm }}$ patients showed a higher level of MCP-1 (20337 \pm 8809 versus $15667 \pm 1611$ versus $54273 \pm 19319 \mathrm{U} ; p<0.05 ; \mathrm{S} 1 / \mathrm{A})$. CCL14 levels did not differ between the study groups $(275613 \pm 36125$ versus $232092 \pm 25276$ versus $320154 \pm 50029 \mathrm{U}$; $p>0.05$; respectively; Figure S1(b)).

\section{Relationship of chemo- and cytokine levels to lung function parameters in all SLE patients}

No association was found between lung function parameters and CCL21 in SLE patients. In contrast, significant association was demonstrated regarding lung function parameters $\mathrm{FVC}$ and $\mathrm{FEV}_{1}$ and IP-10; MCP-1; CCL14, as shown in Figure S2/A-F. Moreover, IP-10 showed correlation with TLC $(r=-0.59 ; p<0.01$; no figure added) and $\operatorname{Raw}(r=0.55 ; p=0.01$; no figure added). In addition, CCL14 correlated negatively with $\mathrm{FEF}_{25-75}(r=-0.69 ; p<0.01$; no figure added), but showed positive correlations with TGV ( $r=0.49 ; p=0.03$; no figure added $), \mathrm{RV}(r=0.54$; $p=0.02$; no figure added $)$ and RV/TLC $(r=0.64$; $p<0.01$; no figure added). 
Table 3 Lung function, diffusion capacity and blood gas parameters of patients with and without lung manifestations

\begin{tabular}{|c|c|c|c|}
\hline Clinical parameters & $\begin{array}{l}\text { SLE patients without } \\
\text { lung manifestations }\end{array}$ & $\begin{array}{l}\text { SLE patients with } \\
\text { lung manifestations }\end{array}$ & $\mathrm{p}$-values \\
\hline \multicolumn{4}{|l|}{ Lung function parameters } \\
\hline FVC (\% pred $)$ & $99.00 \pm 2.75$ & $79.89 \pm 7.26$ & 0.024 \\
\hline FEV1 (\% pred) & $93.11 \pm 3.52$ & $77.00 \pm 7.69$ & 0.075 \\
\hline FEV1/FVC (\% pred) & $101.10 \pm 1.98$ & $96.78 \pm 5.82$ & 0.490 \\
\hline FEF25-75 (\% pred) & $80.67 \pm 9.00$ & $65.22 \pm 11.18$ & 0.297 \\
\hline TLC (\% pred $)$ & $93.22 \pm 3.40$ & $76.89 \pm 3.37$ & 0.003 \\
\hline TGV (\% pred) & $124.60 \pm 4.74$ & $116.30 \pm 7.50$ & 0.368 \\
\hline RV (\% pred) & $95.33 \pm 6.00$ & $89.22 \pm 12.66$ & 0.668 \\
\hline RV/TLC (\% pred) & $100.00 \pm 5.05$ & $111.00 \pm 14.89$ & 0.756 \\
\hline $\operatorname{Raw}(\mathrm{kP} * \mathrm{~s} / \mathrm{L})$ & $0.147 \pm 0.022$ & $0.227 \pm 0.046$ & 0.139 \\
\hline \multicolumn{4}{|l|}{ Diffusion parameters } \\
\hline DLco $(\mathrm{ml} / \mathrm{min} / \mathrm{mmHg})$ & $22.81 \pm 2.06$ & $16.16 \pm 2.13$ & 0.041 \\
\hline DLco $(\%)$ & $90.56 \pm 6.79$ & $60.75 \pm 5.18$ & 0.003 \\
\hline $\operatorname{KLco}(\mathrm{ml} / \mathrm{min} / \mathrm{mmHg})$ & $1.487 \pm 0.10$ & $1.231 \pm 0.09$ & 0.082 \\
\hline KLco $(\%)$ & $72.78 \pm 4.71$ & $60.25 \pm 3.69$ & 0.055 \\
\hline \multicolumn{4}{|l|}{ Blood gas parameters } \\
\hline $\mathrm{pH}$ & $7.449 \pm 0.010$ & $7.456 \pm 0.007$ & 0.551 \\
\hline $\mathrm{pO}_{2}(\mathrm{Hgmm})$ & $83.37 \pm 2.65$ & $91.86 \pm 4.45$ & 0.138 \\
\hline $\mathrm{pCO}_{2}(\mathrm{Hgmm})$ & $32.20 \pm 2.06$ & $30.63 \pm 1.46$ & 0.537 \\
\hline $\mathrm{HCO}_{3}^{-}(\mathrm{mmol} / \mathrm{l})$ & $22.44 \pm 1.38$ & $22.13 \pm 0.77$ & 0.839 \\
\hline \multicolumn{4}{|l|}{ Blood chart results } \\
\hline CRP (mg/L) & $4.62 \pm 1.10$ & $11.24 \pm 4.35$ & 0.385 \\
\hline WBC (G/L) & $7.44 \pm 1.44$ & $9.18 \pm 1.16$ & 0.362 \\
\hline Neutrophil (\%) & $62.99 \pm 3.64$ & $71.86 \pm 4.72$ & 0.159 \\
\hline Thrombocyte (G/L) & $269.40 \pm 26.10$ & $273.90 \pm 38.21$ & 0.923 \\
\hline Lymphocyte (\%) & $26.56 \pm 3.07$ & $20.10 \pm 4.21$ & 0.236 \\
\hline
\end{tabular}

Data expressed as mean \pm SEM if not stated otherwise. Abbreviations are listed in the main text.

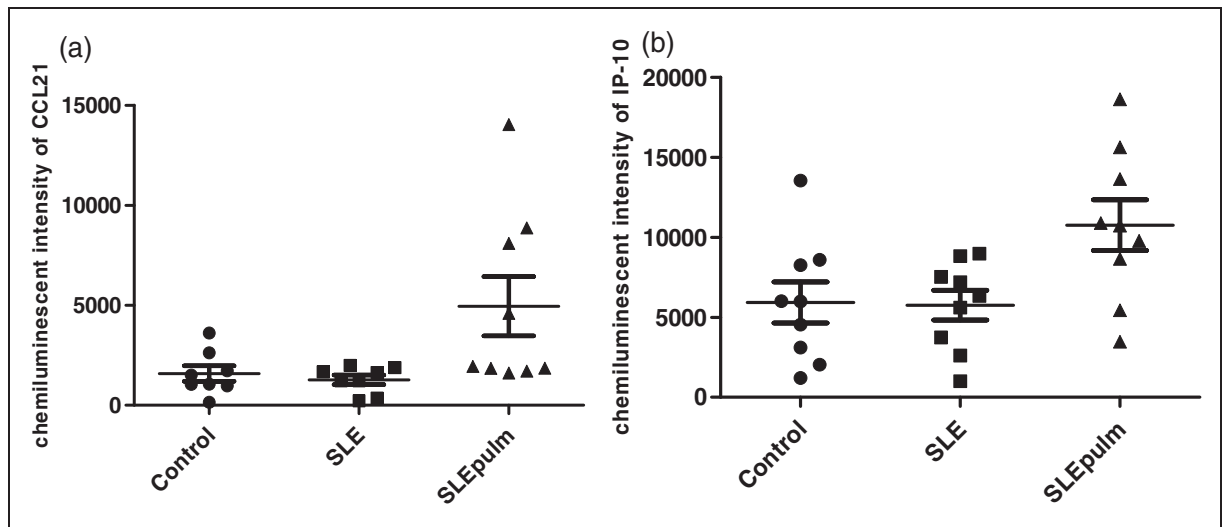

Figure 1 Comparison of CCL21 (a) and IP-10 (b) in healthy volunteers and in systemic lupus erythematosus patients with (SLEpulm) and without pulmonary (SLE) involvement. ((a): SLE versus SLEpulm, $p<0.05$; SLEpulm versus control and SLE, $p<0.05$, respectively).

Relationship of chemo- and cytokine levels to diffusion parameters in all SLE patients

Correlation analyses revealed positive correlation between the chemiluminescent intensity of CCL21 and diffusion parameters, including $\mathrm{DL}_{\mathrm{CO}}(r=$ $-0.73 ; p<0.01$; Figure S3(a)) and $\mathrm{KL}_{\mathrm{CO}}(r=-0.62$; $p<0.01$; Figure S3(b)). However, IP-10; MCP-1 and
CCL14 did not show correlation with diffusion parameters in SLE patients (all $p$-values $>0.05$ ).

ROC analysis of CCL21 and IP-10 values in SLE patients with and without lung manifestations

The potential value of CCL21 and IP-10 in the identification of lung involvement in SLE patients 
was tested using ROC analysis comparing SLE patients without and with pulmonary manifestations. Statistical significance was proved for both CCL21 and IP-10.

ROC analysis of CCL21 values in the SLE patient groups with and without pulmonary involvement yielded an AUC of 0.847 (95\% CI: $0.593-0.972$ ) with a $1664.39 \mathrm{U}$ cut-off value of CCL21 to discriminate between patients with and without pulmonary manifestation (sensitivity \%: 88.90; specificity\%: 75.00; $p<0.01$; Figure S4(a)).

The area under the ROC curve for identification of pulmonary involvement in the case of IP-10 was 0.815 (95\% CI: $0.565-0.955)$. The cut-off value of IP-10 to discriminate between SLE $_{\text {pulm }}$ and SLE without pulmonary manifestations was $8971.62 \mathrm{U}$ (sensitivity\%: 66.67, specificity\%: 100; $p<0.01$; Figure S4(b)).

\section{Discussion}

The search for lupus biomarkers has emerged enormously in the last few years; however, biomarkers for detection of the rare but severe lung involvement are not yet available. ${ }^{18}$

Our results are the first to describe chemokines in peripheral blood as possible biomarkers for lung involvement in SLE patients. CCL21 and IP-10 showed significant changes according to lung involvement in this autoimmune disorder. CCL21 demonstrated strong correlation with decline in diffusion parameters, whereas IP-10 was associated with decreased static lung volumes and lung function parameters indicating airway involvement. Moreover, based on our ROC analysis results, these biomarkers could be promising for the differentiation of pulmonary involvement in SLE patients. MCP-1 did not differ significantly in study groups; however, the correlation results are indicative for further studies about this chemokine as well.

The age of the patients did not differ significantly between our study groups, but it tended to be higher in SLE patients. The age of the individual patients ranged in the same way within the two groups, except for three younger patients in the $\mathrm{SLE}_{\text {pulm }}$ group (as shown in Table 2). Apparently, it is difficult to discuss the topic due to the known disturbances of the immune system in this disease; however, it might not have influence on our results based on the available literature suggesting that age-associated changes in cytokine production are not consistent. ${ }^{19}$
The role of chemokines and cytokines in SLE is extensively investigated due to the known disturbances of the immune system in this disease. Dysregulated activation of both $\mathrm{T}$ and $\mathrm{B}$ lymphocytes and subsequent polyclonal activation of circulating B lymphocytes were shown in previous studies. ${ }^{20}$ These abnormalities lead to the production of a large amount of autoreactive antibodies and the formation of immune complexes responsible for tissue and organ damage. ${ }^{21}$ Chemokines are important for leukocyte recruitment into inflamed tissues, initiation of $\mathrm{T}$ cell immune response and regulation of differential recruitment of $\mathrm{T}$ helper (Th)1 and Th2 lymphocytes. ${ }^{22,23}$ Chemokines are divided into four subfamilies depending on the position of the conserved cysteine residues $(\mathrm{CXC}, \mathrm{CC}, \mathrm{C}$ and $\mathrm{CX} 3 \mathrm{C}) .{ }^{24}$ There has been growing evidence showing that infiltration of $\mathrm{T}$ lymphocytes and other leukocytes into the inflamed tissues might play a critical role in organ involvement in SLE. ${ }^{25}$

CCL21 is a chemotactic chemokine that binds to and activates its receptor - CCR7 - and induces cellular activation and migration. CCR7 is highly expressed on T-lymphocytes and activated dendritic cells, where it has an important role in the navigation of these cells into lymphoid tissues. ${ }^{26} \mathrm{On}$ the other hand, CCL21 and CCR7 have been identified as crucial players in the progression of lung fibrosis. $^{27}$ CCR7 is expressed on lung fibroblasts and is important in their activation, survival and proliferation. $^{28} \mathrm{~A}$ study by Pierce and colleagues demonstrated that CCL21 modulates the functional properties of idiopathic pulmonary fibrosis/ usual interstitial pneumonia fibroblasts. ${ }^{29}$ The role of CCL21 in SLE was not widely investigated; however, its close correlation with diffusion parameters $\mathrm{DL}_{\mathrm{CO}}$ and $\mathrm{KL}_{\mathrm{CO}}$ in our study suggests potential functions in interstitial lung involvement in SLE patients.

The chemokine receptor IP-10 was found significantly up-regulated in our SLE pulm $_{\text {chort. IP-10 is }}$ secreted by several cell types, including $\mathrm{T}$ lymphocytes, neutrophils, monocytes, endothelial cells or fibroblasts. ${ }^{30}$ It is chemotactic for activated Th1 cells and together with IL-6 induces plasma cell differentiation. ${ }^{31}$ Recruited Th1 lymphocytes may be responsible for enhanced IFN- $\gamma$ and TNF- $\alpha$ production, which in turn stimulates IP-10 secretion from a variety of cells, leading to an amplification feedback loop. The importance of these substances was demonstrated as elevated levels of IFN- $\gamma$ and TNF- $\alpha$ were found in SLE patients with pulmonary involvement. $^{32,13}$ 
IP-10 level was elevated in the serum, urine and cerebrospinal fluid of SLE patients depending on the level of disease activity. ${ }^{33,34}$ Our data also suggest that higher IP-10 values are associated with more pronounced respiratory involvement; however, data on disease activity score were not available. CC chemokines are also important in inflammatory cell recruitment into the lungs in idiopathic interstitial pneumonias. ${ }^{35,36}$ Although blood levels of IP-10 have not been widely investigated in patients with lung fibrosis, some studies suggest their role in other fibrotic diseases (e.g. liver fibrosis), where elevated levels of IP-10 were detected. ${ }^{37}$ Antonelli and colleagues demonstrated that high IP-10 values are associated with more severe clinical phenotype and with the presence of lung fibrosis in systemic sclerosis patients. ${ }^{38}$

Markers of inflammatory activity are important for the assessment and management of chronic airway diseases with chronic inflammation and loss of lung volume, like chronic obstructive pulmonary disease (COPD) ${ }^{39}$ IP-10 was elevated in exacerbated COPD patients' sputum and another study showed negative correlation with FVC. ${ }^{40,41}$ Moreover, the marker increased from baseline in human rhinovirus induced exacerbations and correlated with sputum viral load in COPD subjects. ${ }^{42}$ IP-10 is supposed to have a central role in Th1 mediated diseases. ${ }^{43}$ Consistent with that, our research group has previously described that SLE patients with pulmonary involvement had significant changes in peripheral Th1/Th2 cells. ${ }^{5}$ The findings in COPD patients and our lung function $\left(\mathrm{FEV}_{1}\right.$ and $\left.\mathrm{FVC}\right)$ correlation results suggest that IP-10 in SLE patients with lung involvement can be an indicator of inflammatory activation in the airways and loss of lung function in SLE patients.

MCP-1 also showed correlation with lung function parameters $\left(\mathrm{FVC}(\%)\right.$ and $\left.\mathrm{FEV}_{1}(\%)\right)$ and showed a tendency for up-regulation in patients with pulmonary involvement. Many studies have found correlation between disease activity and MCP-1, mostly in urinary samples indicating the severity of lupus nephritis. ${ }^{44}$ Moreover, it has been described as a potential biomarker in the prediction of future flare of SLE. ${ }^{45}$ However, to our knowledge, its role in lung involvement has not been investigated. Like IP-10, MCP-1 is also a proinflammatory cytokine which can play a role in the inflammatory activation of the airways, contributing to the development of respiratory symptoms. On the other hand, CCL14 also correlated negatively with lung function parameters $\left(\mathrm{FVC}(\%)\right.$ and $\left.\mathrm{FEV}_{1}(\%)\right)$; however, it showed no difference in the two study groups. It would be important to investigate these biomarkers on a larger study cohort to establish them as a possible diagnostic marker of lung involvement in SLE.

\section{Conclusions}

Lung involvement in SLE is rare, but often severely influences patient outcome. Our results suggest that IP-10 and CCL21 might be important biomarkers in the differentiation of SLE patients with and without pulmonary involvement. Our patients showed similar respiratory symptoms, and only lung function was significantly different between the groups. As these patients are prone to pulmonary symptoms, it is of major interest if pulmonary changes that cannot be measured clinically could be predicted using IP-10 and/or CCL21. CCL21 seems to be mainly a marker for interstitial lung involvement, while IP-10 is more a marker for lung volume and airway centred lung changes of SLE. The importance of our work is in identifying significant and important blood biomarkers for possible use as an easy test in clinical praxis for this rare condition; however, our results need to be confirmed on a larger study population.

\section{Declaration of conflicting interests}

The author(s) declared no potential conflicts of interest with respect to the research, authorship, and/or publication of this article.

\section{Funding}

The author(s) disclosed receipt of the following financial support for the research, authorship, and/or publication of this article: The study was supported by Hungarian National Research Fund (OTKA 68808) and Hungarian Respiratory Society (grant to Andras Bikov). This publication was supported by the Janos Bolyai Research Scholarship of the Hungarian Academy of Sciences to Andras Bikov.

\section{References}

1 Choi J, Kim S, Craft J. The pathogenesis of systemic lupus erythematosus - an update. Curr Opin Immunol 2012; 24: 651-657.

2 Costenbader K, Feskanich D, Stampfer M, Karlson E. Reproductive and menopausal factors and risk of systemic lupus erythematosus in women. Arthritis Rheum 2007; 56: 1251-1262. 
3 Danchenko N, Satia JA, Anthony MS. Epidemiology of systemic lupus erythematosus: a comparison of worldwide disease burden. Lupus 2006; 15: 308-318.

4 Rahman A, Isenberg D. Systemic lupus erythematosus. $N$ Engl $J$ Med 2008; 358: 929-939.

5 Vincze K, Kovats Z, Cseh A, et al. Peripheral CD4+ cell prevalence and pleuropulmonary manifestations of systemic lupus erythematosus patients. Respir Med 2014; 108: 766-774.

6 Alamoundi O, Attar SM. Pulmonary manifestations in systemic lupus erythematosus: Association with disease activity. Respirology 2015; 20: 474-480.

7 Trapani S, Camiciottoli G, Ermini M, Castellani W, Falcini F. Pulmonary involvement in juvenile systemic lupus erythematosus: a study on lung function in patients asymptomatic for respiratory disease. Lupus 1998; 7: 545-550.

8 Pines A, Kaplinsky N, Olchovsky D, Rozenman J, Frankl O. Pleuropulmonary manifestations of systemic lupus erythematosus: clinical features of its subgroups. Prognostic and therapeutic implications. Chest 1985; 88: 129-135.

9 Torre O, Harari S. Pleural and pulmonary involvement in systemic lupus erythematosus. Presse Med 2011; 40: e19-29.

10 Allen D, Fischer A, Bshouty Z, et al. Evaluating systemic lupus erythematosus patients for lung involvement. Lupus 2012; 21: $1316-1325$.

11 Memet B, Ginzler EM. Pulmonary manifestations of systemic lupus erythematosus. Semin Respir Crit Care Med 2007; 28: 441-450.

12 Adhya Z, Borozdenkova S, Karim MY. The role of cytokines as biomarkers in systemic lupus erythematosus and lupus nephritis. Nephrol Dial Transplant 2011; 26: 3273-3280.

13 Al-Mutairi S, Al-Awadhi A, Raghupathy R, et al. Lupus patients with pulmonary involvement have a pro-inflammatory cytokines profile. Rheumatol Int 2007; 27: 621-630.

14 Nielepkowicz-Goździńska A, Fendler W, Robak E, et al. Exhaled cytokines in systemic lupus erythematosus with lung involvement. Pol Arch Med Wewn 2013; 123: 141-148.

15 Yoshio T, Masuyama JI, Kohda N, et al. Association of interleukin 6 release from endothelial cells and pulmonary hypertension in SLE. J Rheumatol 1997; 24: 489-495.

16 Hochberg M. Updating the American College of Rheumatology revised criteria for the classification of systemic lupus erythematosus. Arthritis Rheum 1997; 40: 1725-1734.

17 Miller MR, Hankinson J, Brusasco V, et al. Standardisation of spirometry. Eur Respir J 2005; 26: 319-338.

18 Herbst R, Liu Z, Jallal B, Yao Y. Biomarkers for systemic lupus erythematosus. Int $J$ Rheum Dis 2012; 15: 433-444.

19 Bernstein ED, Murasko DM. Effect of age on cytokine production in humans. Age 1998; 21: 137-151.

20 Wong CK, Lit LCW, Tam LS, Li EK, Lam CWK. Aberrant production of costimulatory molecules CTLA-4, CD28, CD80 and CD86 in patients with systemic lupus erythematosus. Rheumatology 2005; 44: 989-994.

21 Heinlen LD, McClain MT, Merrill J, et al. Clinical criteria for systemic lupus erythematosus precede diagnosis, and associated autoantibodies are present before clinical symptoms. Arthritis Rheum 2007; 56: 2344-2351.

22 Premack BA, Schall TJ. Chemokine receptors: gateways to inflammation and infection. Nat Med 1996; 2: 1174-1178.

23 Sallusto $\mathrm{F}$. The role of chemokines and chemokine receptors in $\mathrm{T}$ cell priming and Th1/Th2-mediated responses. Haematologica 1999; 84: 28-31.

24 Luster AD. Chemokines - chemotactic cytokines that mediate inflammation. $N$ Engl J Med 1998; 338: 436-445.

$25 \mathrm{Yu}$ SL, Kuan WP, Wong CK, Li EK, Tam LS. Immunopathological roles of cytokines, chemokines, signaling molecules and pattern-recognition receptors in systemic lupus erythematosus. Clin Dev Immunol 2012; DOI: 10.1155/2012/ 715190. Epub 23 January 2012.

26 Comerford I, Harata-Lee Y, Bunting MD, Gregor C, Kara EE, McColl SR. A myriad of functions and complex regulation of the CCR7/CCL19/CCL21 chemokine axis in the adaptive immune system. Cytokine Growth Factor Rev 2013; 24: 269-283.

27 Trujillo G, Hartigan AJ, Hogaboam CM. T regulatory cells and attenuated bleomycin-induced fibrosis in lungs of CCR7-/- mice. Fibrog Tissue Repair 2010; 3: 18.

28 Habiel DM, Hogaboam C. Heterogenity in fibroblast proliferation and survival in idiopathic pulmonary fibrosis. Front Pharmacol 2014; 5: 1-6.

29 Pierce EM, Carpenter K, Jakubzick C, et al. Idiopathic pulmonary fibrosis fibroblasts migrate and proliferate to CC chemokine ligand 21. Eur Respir J 2007; 29: 1082-1093.

30 Antonelli A, Ferrari SM, Giuggioli D, Ferrannini E, Ferri C, Fallahi P. Chemokine (C-X-C motif) ligand (CXCL) 10 in autoimmune diseases. Autoimmun Rev 2014; 13: 272-280.

$31 \mathrm{Xu} \mathrm{W}$, Joo H, Clayton S, et al. Macrophages induce differentiation of plasma cells through CXCL10/IP-10. J Exp Med 2012; 209: $1813-1823$

32 Antonelli A, Ferri C, Ferrari SM, Colaci M, Fallahi P. Immunopathogenesis of $\mathrm{HCV}$ related endocrine manifestations in chronic hepatitis and mixed cryoglobulinemia. Autoimmun Rev 2008; 8: 18-23.

33 Narumi S, Takeuchi T, Kobayashi Y, Konishi K. Serum levels of IFN-inducible protein-10 relating to the activity of systemic lupus erythematosus. Cytokine 2000; 12: 1561-1565.

34 Abujam B, Cheekatla S, Aggarwal A. Urinary CXCL-10/IP-10 and MCP-1 as markers to assess activity of lupus nephritis. Lupus 2013; 22: 614-623.

35 Yoshioka S, Mukae H, Sugiyama K, et al. High-BAL fluid concentrations of RANTES in nonspecific interstitial pneumonia compared with usual interstitial pneumonia. Respir Med 2004; 98: $945-51$.

36 Nakayama S, Mukae H, Ishii H, et al. Comparison of BALF concentrations of ENA-78 and IP-10 in patients with idiopathic pulmonary fibrosis and nonspecific interstitial pneumonia. Respir Med 2005; 99: 1145-1151.

37 Sevgi DY, Bayraktar B, Gündüz A, et al. Serum soluble urokinase activator receptor and interferon-?-induced protein 10 levels correlate with significant fibrosis in chronic hepatitis B. Wien Klin Wochenschr 2015; DOI: 10.1007/s00508-015-0886-4.

38 Antonelli A, Ferri C, Fallahi P, et al. CXCL $10(\alpha)$ and CCL2 ( $\beta)$ chemokines in systemic sclerosis - a longitudinal study. Rheumatology 2008; 47: 45-49.

39 Taylor DR, Pavord ID. Biomarkers in the assessment and management of airways diseases. Postgrad Med J 2008; 84: 628-634.

40 Warwick G, Thomas PS, Yates DH. Non-invasive biomarkers in exacerbations of obstructive lung disease. Respirology 2013; 18: 874-884.

41 Eickmeier O, Huebner M, Herrmann E, et al. Sputum biomarker profiles in cystic fibrosis $(\mathrm{CF})$ and chronic obstructive pulmonary disease (COPD) and association between pulmonary function. Cytokine 2010; 50: 152-157.

42 Quint JK, Donaldson GC, Goldring JJ, Baghai-Ravary R, Hurst JR, Wedzicha JA. Serum IP-10 as a biomarker of human rhinovirus infection at exacerbation of COPD. Chest 2010; 137: 812-822.

43 Jinquan T, Jing C, Jacobi HH, et al. CXCR3 expression and activation of eosinophils: role of IFN-gamma-inducible protein-10 and monokine induced by IFN-gamma. J Immunol 2000; 165: $1548-1556$.

44 Li Y, Tucci M, Narain S, et al. Urinary biomarkers in lupus nephrtis. Autoimmun Rev 2006; 5: 383-388.

45 Bauer JW, Petri M, Batliwalla FM, et al. Interferon-Regulated as Biomarkers of Systemic Lupus Erythematosus Disease Activity: A Validation Study. Arthritis Rheum 2009; 60: 3098-3107. 